\title{
From coal-face clinicians to change agents - igniting healthcare innovation
}

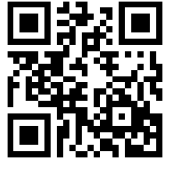

Two young Cape Townbased doctors, evoked by the overwhelm they've seen at struggling public hospitals, have custom-educated themselves to facilitate pioneering, inclusive healthcare solutions, bringing local patients, healthcare workers and technicians together for the first time.

Capetonian Dr Francois Bonnici, who founded the Bertha Centre for Social Innovation and Entrepreneurship (Bertha Centre) at the University of Cape Town (UCT)'s Graduate School of Business (GSB), and Pretoria-born, Dr Lindi van Niekerk, who recently joined him, have just put creative solutions - be they high-tech, low-tech or 'no-tech' - at the centre of South Africa (SA)'s healthcare radar. South Africans are known globally for creative, highly appropriate and effective custombuilt solutions in healthcare, ranging from wind-up Doppler ultrasound fetal heart rate monitors, home-based parental care for young tracheotomy patients, to containerbased HIV care for truckers and sex workers on our highways. However, this broad ingenuity has never been formally embraced or framed in an inclusive, philanthropic way - or given a solid platform for expression.

Bonnici and Van Niekerk are doing just that - with two successful launch events held in Cape Town at the end of January; the first, a 2-day Healthcare Hackathon, at Groote Schuur Hospital (GSH). Here 60 of 100 shortlisted applicants, including healthcare workers, students, software developers, engineers, entrepreneurs and designers, collaborated and competed in teams to produce a workable prototype - matched with a sustainable business model for showcasing countrywide - with a R15 000 prize up for grabs for the most innovative, practical outcome.

The second event, also in Cape Town and a few days later, was an inclusive and carefully thought out Healthcare Innovation Summit, to which a broad but select sweep of delegates, mainly local, but many from across the continent, were invited. Aimed at addressing Africa's most pressing healthcare needs, topics included 'collaboratively re-imagining healthcare, opportunities for healthcare innovation, better healthcare design, transforming primary care from

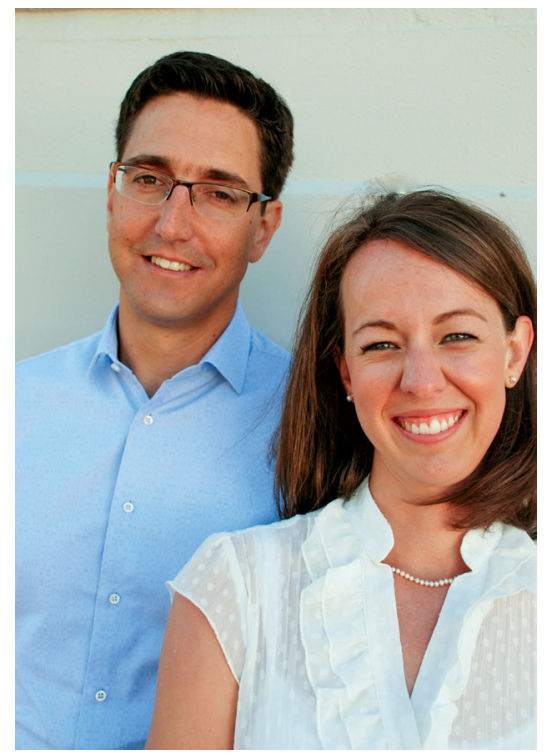

Healthcare game-changers Drs Francois Bonnici and Lindi van Niekerk. Picture: Chris Bateman.

the inside out, navigating the innovation ecosystem and assessing innovation impact'. The centre has also published a Health Innovators Review featuring leading health innovators in SA who have successfully implemented inclusive, effective and affordable solutions that have improved care.

A quick assessment of Bonnici and Van Niekerk's own impact after they ran headlong into major delivery challenges during their public hospital tenures makes for impressive reading and underscores the philanthropic zeal with which they've set about their task.

\section{When the personal \\ becomes pragmatic and political}

Bonnici graduated top of his UCT Medical School class and his evolution includes a Rhodes scholarship (he was top of his local MBA class and completed a Public Health degree at Oxford), the Namibian public health sector, Mowbray Maternity Hospital (community service), and some paediatric specialisation at Cape Town's Red Cross War Memorial Children's Hospital. He left Red Cross to hone his newly acquired business management and social innovation skills in Geneva and Davos in Switzerland, working for both the Global Fund for HIV/TB and Malaria and the World Economic Forum where he says he 'got to understand the top-end, 30000 foot stuff'. Public/private partnerships were an integral part of his overseas work sojourns. Stints at earthquake disasters in India and public child health in Mexico reaffirmed his formative SA/ Namibian experiences.

"My time in Geneva made me think that the top-down solutions were so far removed from what I was passionate about - so I began looking at things from the bottom-up. Finding new ways of creatively overcoming challenges and constraints - whether private business modalities or public ones,' he explained. Among his collaborative creations is the wind-up, low-cost fetal heart rate and oxygen monitor which won the global Index Design for Life award and which is now used by Médecins Sans Frontiéres in 15 countries. Bonnici 'walked the walk' well before Oxford; this included a 1999 stint at Windhoek's Katutura State Hospital where he 'lived with, and learnt everything from the nurses,' admitting newborn infants in cardboard boxes or wrapped in newspaper and colliding headfirst with a $16 \%$ premature birth rate. He found babies stacked three to an incubator (the hospital had eight of these). 'That year we did 45 resuscitations - 44 were unsuccessful,' he recalls. Newborn health was 'under-recognised' at the time as a cause of infant mortality, says Bonnici, so he canvassed the opinion of Katutura Hospital's initially sceptical paediatrician (one of three in Namibia at the time) and nurses, intent on introducing a Kangaroo Care (KC) programme. He sat down and wrote a KC policy, which was forwarded to Namibia's Minister of Health. Within four months it became official Namibian health policy. Immediately after this and during his community service at Mowbray Maternity Hospital (which he called his 'night job'), he realised he could again add more value. 'I wrote to the province and told them about my public health degree, so my day job turned into improving newborn care at 26 secondary urban and rural hospitals.' Mowbray Maternity has a special place in his heart; he was born there and met his wife there. The first concrete underpinning of his passion for innovation came after his return from Geneva when he approached UCT's GSB to begin a Centre for Social Innovation and Entrepreneurship. 'There 
was so much happening in SA, but all our top campus researchers were overseas. UCT was positive, but had no spare money and [we] were fortunate enough to find a South African family foundation in London' (which began the Bertha Foundation). The Bertha Foundation funds activism, whether it is Corruption Watch, Equal Education, the non-governmental organisation Section 27 , or documentaries on societal issues. Today the GSB's Bertha Centre works across disciplines. Bonnici spends a significant amount of time with National Treasury applying his skills to social financing while helping build socially motivated businesses.

\section{Converting deep frustration into helpful compassion}

During Lindi van Niekerk's internship at Victoria Hospital she saw patients dying while awaiting a place in GSH's dialysis queue, which spurred her on to try to make a difference. 'There were multiple cases every day of patients being turned away. The realisation dawned on me that we could still do something, even if we weren't curing.' So, she pioneered the Abundant Life care programme that helps the Hospice and Palliative Care Association in the Cape Metropole to handle the overflow from its maximum caseload of 800 patients per annum.

Van Niekerk adds, 'Patients are dying of organ failure because we don't have the resources, and one in five patients will need end-of-life care in our hospitals'. Abundant Life's main aim is to get patients supporting one another and back home, delivering wheelchairs, bedpans and other vital equipment to make this possible. Her programme, supported by Rotary (funding for pain management), GSH and various day hospitals, has reduced admissions by about $40 \%$ and increased managed deaths at home in the Cape Metropole by between $60 \%$ and $70 \%$. She first met Bonnici while at Victoria Hospital and decided to do public health. 'My consultants said nobody wants to do that!' she laughs. However, after 'exploring different places where I could bring about change, she put her ambition of becoming a neurologist on hold, a 2010/2011 scholarship to study public health at the London School of Tropical Medicine, the welcome interruption.

Like Bonnici, she realised how quickly a physician could become 'detached from the realities on the ground,' and convinced the province to let her do palliative care work during her community service tenure. 'I was driven by my own "failures" within the system. I wanted to make a difference and really do something for people', she adds. The GSB Bertha Centre is the first of its kind in SA and the Bonnici/Van Niekerk duo have crafted their own job descriptions. 'It's a unique opportunity. We're extremely fortunate to be involved and it's very different to what my friends are doing,' says Van Niekerk. The pair believe that as clinicians, it's 'easy to lose the bigger picture perspective, especially where it links to other disciplines and what we can learn and bring in'

Put differently, and to quote one of the Eastern Cape's award-winning deep rural hospital delegates to the Innovation Summit, Zithulele Hospital's Chief Medical Officer, Dr Ben Gaunt, 'if you don't have a dream, how will you ever have a dream come true?'

\section{Chris Bateman}

chrisb@hmpg.co.za

S Afr Med J 2014;104(3):159-160.

DOI:10.7196/SAMJ.7997 